\title{
Discontinuity patterns in stages of the precaution adoption process model: Meat consumption during a livestock epidemic
}

\author{
Falko F. Sniehotta', Aleksandra Luszczynska ${ }^{2 *}$, Urte Scholz $^{3}$ and \\ Sonia Lippke 3 \\ 'University of Aberdeen, UK \\ ${ }^{2}$ Warsaw University, Poland \\ ${ }^{3}$ Freie Universitaet Berlin, Germany
}

\begin{abstract}
Objectives. Patterns of changes in social-cognitive variables were investigated in order to test selected stages of the precaution adoption process model (PAPM). It was hypothesized that non-linear trends (discontinuity patterns) in perceived vulnerability, positive and negative outcome expectancies, procrastination, and self-efficacy might be observed across the stages.
\end{abstract}

Design. Cross-sectional data from 808 respondents were employed.

Method. Questionnaire data were collected in an on-line study on meat consumption during a livestock epidemic in Germany. Polynomial trends and analyses of variance with post-hoc-contrasts were used to examine the patterns of change.

Results. Discontinuity patterns were found for perceived vulnerability, negative outcome expectancies, and procrastination. The data provided partial support for discontinuity patterns in self-efficacy. Continuity patterns were found for positive outcome expectancies.

Conclusions. Results provide support for a stage model rather than a pseudo-stage model.

Bovine spongiform encephalopathy (BSE) is a lethal disease in cattle caused by prions (Prusiner, 1997). The transmission of BSE causes a new variant of Creutzfeld-Jacob disease in mice (Bruce et al., 1997). The new variant of Creutzfeld-Jacob disease is a rare but lethal nervous disease that also occurs in human beings. Health risks of possible BSE infection and its consequences became an emerging issue in medical studies (Ferguson, Ghani, Donelly, Hahenaars, \& Anderson, 2002) and in news headlines in German mass media. After the detection of the first case of BSE in Germany in November 2000,

\footnotetext{
* Correspondence should be addressed to Aleksandra Luszczynska, University of Sussex, Dept of Psychology, Falmer BNI 9QH, UK (email: aluszczynska@sussex.ac.uk) or Falko F. Sniehotta, School of Psychology, College of Life Sciences and Medicine, William Guild Building, University of Aberdeen, Aberdeen AB24 2UB, Scotland, UK (email: f.sniehotta@abdn.ac.uk).
} 
a serious decrease in beef consumption was observed (German Central Agency for Market and Price Reports [GCAMPR], 2001). On the background of daily reports about BSE and other health risks such as food additives (e.g. antibiotics) or foot and mouth disease that dispersed over Europe in the spring of 2001, meat consumption decreased. For example, in April 2001, 38\% less beef was sold compared with the previous year (GCAMPR, 2001). During the BSE epidemic, the perceived risk of disease related to meat consumption led to precautionary actions, and a reduction of meat consumption (Weitkunat et al., 2003).

Using reduction of meat consumption during a livestock epidemic as an example, the present study examines the assumption that health behaviour changes in distinct stages. Stages from the precaution adoption process model (PAPM; Weinstein, 1988) were examined along with two additional stages. Change patterns in social-cognitive variables, such as perception of vulnerability, outcome expectancies, procrastination and self-efficacy were tested.

\section{Stage models}

The basic assumption of stage models is that behaviour change is a process that can be divided into distinct stages. For example, these stages describe the development of an intention to change, initiation and maintenance of a health behaviour and, finally, recovery after lapses. Whereas models such as the PAPM (Weinstein, 1988) provide seven stages, other approaches such as the health action process approach (HAPA; Schwarzer, 2001) propose only two stages. Some stages of the PAPM are similar to those of the transtheoretical model (TTM; Prochaska \& DiClemente, 1982). In contrast to stage models, continuum models such as the theory of planned behaviour (Ajzen, 1991) assume no distinction of the process of change into stages. In continuum models, the likelihood of a specific health behaviour can be predicted from a given set of attitudes, competencies, and beliefs. Thus, a change in these constructs corresponds with behaviour change. Researchers who emphasize the utility of continuum models suggest that the distinction between stages is arbitrary and in most cases not sufficiently based on empirical evidence (Sutton, 1998).

\section{Testing stage models}

There are three approaches to test stage models: the analysis of cross-sectional data, the prediction of stage transitions in longitudinal designs, and experimental approaches (Sutton, 2000; Weinstein, Rothman, \& Sutton, 1998). The analysis of cross-sectional data is most frequently used to test stage models. This approach allows testing a number of hypotheses on cross-sectional differences between stages for several variables.

Sutton (2000) proposed a methodological framework for testing the assumptions of stages using data in cross-sectional designs. In this approach, support for a stage model requires more than significant between-groups differences in the levels of a selected variable. If the subgroups of participants differ significantly regarding a relevant variable, and a consecutive linear effect across the stages is observed, then such a result shows a continuity pattern. Therefore, it can be assumed that the fragmentation into stages is unnecessary and misleading. The artificial fragmentation of a continual linear process into stages results in pseudo-stages (Sutton, 2000). In pseudo-stages, the same variable equally influences the progression from one stage to another. Discontinuity patterns would support the assumptions of a stage model. If a level of a relevant variable 
changes across stages in a way different from a consequent increase or decrease across the stages, the influence of this variable for the stage progression is likely to be dependent on the stage (Sutton, 2000).

\section{The precaution adoption process model (PAPM)}

The PAPM explains the processes of adoption of risk-reducing behaviours. Previous applications of the model have addressed other preventive health behaviours such as radon home testing (Weinstein, Lyon, Sandman, \& Cuite, 1998), osteoporosis prevention (Blalock et al., 1996), and mammography screening (Clemow et al., 2000). The PAPM includes seven stages (Weinstein \& Sandman, 1992). Stage 1 (unaware of issue) describes a state in which a person is not aware of a potential health threat or a protective behaviour. A stage of unawareness is specific to the PAPM and it has not been studied sufficiently (Weinstein, 1988). If an individual is aware of the issue but not personally engaged, then Stage 2 (unengaged by issue) is reached. Stage 3 (deciding about action) is characterized by deliberations about costs and benefits. This stage is similar to the contemplation phase, described in the TTM. If this decision is negative, the person abandons the process of change and reaches Stage 4 (decided not to act). If the decision is positive, the person forms an intention and progresses from Stage 3 to Stage 5 (decided to act). The two action stages of the PAPM are similar to the ones in the TTM (Weinstein \& Sandman, 1992). Stage 6 (acting) refers to initiation of the action, and Stage 7 (maintenance) describes additional stabilization of initiated actions. According to the assumptions of the PAPM, successful behaviour change requires that an individual passes through the stages in a sequence of $1,2,3,5,6,7$, without skipping a stage.

Some individuals initiate a new behaviour and maintain it. However, not everyone who initiates the action progresses to the maintenance stage. Some individuals might consider their behaviour change to be temporary. Those who reduce their meat consumption due to information about BSE and foot and mouth disease might have no long-term intentions for maintenance. These might be finally formed after receiving further information about the progress in the epidemic range. Therefore, in the present study, a new distinction between Stages 6a (acting with no intention of maintenance) and $6 \mathrm{~b}$ (acting with intention of maintenance) has been introduced. The maintenance stage (acting for more than 6 months) has not been included because the duration of the livestock epidemic was shorter than 6 months when the data collection was started, which is the crucial time period for the assignment to the maintenance stage according to the PAPM.

\section{The role of social-cognitive variables across the stages}

The PAPM does not provide a fixed set of key variables that differentiate between stages or foster progression from stage to stage. According to many models of health behaviour change, such as HAPA or TTM, social-cognitive variables like perceived vulnerability, outcome expectancies about precautionary actions such as changing one's meat consumption, and self-efficacy beliefs about changing one's meat consumption might influence the change process. In the present study, it was hypothesized that these variables allow for differentiating between the selected stages of the PAPM.

Perceived vulnerability to develop a disease due to meat consumption might have an influence in the early stages of decision making (Schwarzer, 1999; Weinstein, 1988). 
People who think about taking precautionary actions should perceive higher vulnerability than those who are unengaged (Clemow et al,, 2000). Taking precautionary action reduces the actual risk and, as a consequence, the perceived risk. Positive outcome expectancies of an adjusted diet (i.e. low meat consumption) are assumed to increase over the PAPM stages. Individuals who decide not to act might see less beneficial outcomes than those deciding what to do or those who have already decided to act (Clemow et al., 2000; Weinstein \& Sandman, 1992). Negative outcome expectancies might be more salient in pre-decisional stages than in post-decisional and action stages (Lee, 1993). Nevertheless, when people take action they perceive fewer barriers (Blalock et al., 1996). Perceived self-efficacy might be more important in later stages, after an individual has developed an intention (Schwarzer, 2001). If one is not aware of the issue or is not engaged, self-efficacy should not play a significant role. A regular increase of self-efficacy across the PAPM stages has been found for osteoporosis prevention (e.g. Blalock et al., 1996).

Additionally, changes in patterns of procrastination over the stages have been examined. Procrastination is the tendency to delay decisions and the execution of intended actions (Tice \& Baumeister, 1997). A lack of self-regulatory competence can affect different behavioural domains. Thus, strong procrastination should be typical for individuals in Stage 3 (deciding) and Stage 5 (decided to act, but not acting yet) of the PAPM. In a study on mammography utilization the highest procrastination was found in Stage 5, decided, but not acting yet (Clemow et al., 2000).

\section{Research questions}

The present study examined differences in social-cognitive variables between stages of health behaviour change in the context of a prevention behaviour, low meat consumption. Five stages of the PAPM and two additional stages of acting with and without intention to maintain the behaviour were explored. The question was whether discontinuity patterns in social-cognitive variables might emerge over the stages, that is, whether these variables were high in selected stages while being low in other stages. The following patterns were hypothesized: (1) low vulnerability in unengaged (Stage 2) respondents, followed by high vulnerability across the decision stages and low vulnerability in action stages, especially in respondents acting with the intention to maintain, (2) increase of pros (positive outcome expectancies) across the stages, with low pros in Stage 4, decided not to act, (3) increase of cons (negative outcome expectancies) across the stages up to Stage 5, decided to act, followed by low cons at the action stages, with low cons in Stage 4 (decided not to act), (4) low self-efficacy in predecisional and decisional stages, especially in stages that apply to being stuck in the decision process (deciding and decided not to act), followed by high self-efficacy in action stages, especially in respondents acting with the intention to maintain, (5) low procrastination in those who are unengaged or acting and high in those who are deciding or decided to act but who procrastinate to change their behaviours.

\section{Method}

\section{Procedure and sample}

An on-line study was conducted in April 2001, during the livestock epidemic in Germany. The study was advertised on Internet websites, such as a website of a German 
university and a website of a popular German weekly magazine. After clicking the advertisement, the Internet users were linked to an invitation to take part in the study. The invitation informed visitors to the website about the research and assured confidentiality. After reading the invitation, those Internet users who marked that they agreed to participate in the study were referred to a web page of the Health Psychology Department of the Free University, Berlin. This web page included instructions about how to answer the questions and the measurements. After reading the instructions, they followed a link to a self-administered questionnaire. Provision of an e-mail address was not a condition of participation in the study. The recruitment of participants was performed in line with ethics standards suggested by the American Association for the Advancement of Science (1999).

A total of 808 individuals ( $49 \%$ were men) completed the measurement. They were from 16 to 64 years old $(M=31.97, S D=10.30)$. Most of the respondents had completed college $(77.1 \%)$ or had obtained a university degree $(38.9 \%)$.

Discontinuity patterns were examined by means of polynomial-based orthogonal contrasts using ANOVA (see Armitage \& Arden, 2002). Strong linear trends can be interpreted as supporting a pseudo-stage model. If quadratic or cubic trends are stronger than linear trends, such a result might be interpreted as an evidence for discontinuity. Trend analyses might be insensitive to some discontinuity in sub-sequences (e.g. when discontinuity in three consecutive stages might be observed), if the pattern in all analysed stages shows strong linearity. Post-boc-contrast analyses were calculated to test the differences between stages.

\section{Measures}

Vulnerability was assessed by three items $(\alpha=.93)$, 'Compared with other persons of your age and gender, how do you estimate the likelihood that you will ever (a) get the Creutzfeldt-Jacob disease associated to BSE, (b) get sick from food additives or medicine used in the mast, (c) be infected by some other animal disease?' The responses for vulnerability were given on 7-point scales, anchored at much below average and much above average.

Fifteen items were used to measure outcome expectancies. The stem, 'What would be the personal consequences for you if you reduced the consumption of meat or continued not to eat meat?', was followed by possible pros ( 8 items, $\alpha=.81$ ), such as, 'I would be less vulnerable to diseases', and cons $(\alpha=.81)$, such as, 'I would feel constricted in my nutrition.' The responses ranged from not at all true (1) to exactly true (4).

The self-efficacy scale was composed of 15 items $(\alpha=.95)$. Participants were asked to report how certain they were that they could overcome certain difficulties that might arise during attempts of adjustment of meat consumption. The stem, 'I'm able to reduce the consumption of meat,' was followed by specific barriers such as '. . .even if it takes several times before I succeed.' The answers scored on a scale ranging from not at all true (1) to exactly true (4).

Procrastination was measured by a 10-item scale $(\alpha=.82)$ developed by Schwarzer, Schmitz, and Diehl (2000). Items referred to the delay of action (e.g. 'I often take on things which I then end up not doing'). The responses ranged from not at all true (1) to exactly true (4).

To assess the stages participants were asked, 'Which of the following subjects have you heard of yet?'. The question was followed by two diseases: (a) 'BSE or Mad Cow 
Disease,' and (b) 'Creutzfeldt-Jacob disease.' The responses were scored on a yes-no scale. Participants who had heard of neither BSE nor Creutzfeldt-Jacob disease were assigned to Stage 1 (unaware of the issue). Respondents who answered positively to that question were asked, 'Have you thought about changing your meat consumption, eating less meat, eating no meat at all, or only certain types of meat? Please read the following eight statements carefully and select the one that fits you best.' This stem was followed by eight items referring to the stages of the PAPM, 'I never thought about changing my meat consumption' (representing Stage 2), 'I am undecided about changing my meat consumption' (Stage 3), 'I have decided that I didn't want to change my meat consumption' (Stage 4), 'I have decided that I wanted to change my meat consumption, but I had not started yet' (Stage 5), 'I have decided that I wanted to change my meat consumption and I had already had my first tries' (Stage 5), 'I have tried to change my meat consumption but I reversed to my prior diet' (Stage 5), 'I changed my meat consumption preliminarily, but I am undecided about the future' (Stage 6a), and 'I changed my meat consumption and I want to keep it like this in the future' (Stage 6b).

All participants in Stage 5 had decided to change their meat consumption and reported that they did not act in accordance with this decision. They had either not yet tried, tried to act, or tried to act and failed. This means that although they differed in their attempts to change their meat consumption, all of them had decided to act, but were not acting and can therefore be assigned to Stage 5 of the PAPM.

\section{Results}

Respondents were assigned to the stages used in the present study according to their answers to the questions of the stage measure. The distribution pattern represents the situation in Germany in April 2001. None of participants was assigned to Stage 1 (unaware of the issue), $62(7.7 \%)$ participants were in Stage 2 (unengaged by issue), 63 (7.8\%) in Stage 3 (deciding), 121 (15.0\%) in Stage 4 (decided not to act), 64 (7.9\%) in Stage 5 (decided to act), $186(23.0 \%)$ in Stage 6a (acting with no intention about maintenance) and 312 (38.6\%) in Stage 6b (acting with intention about maintenance).

To test whether participants assigned to Stage 5 form one group or whether this group should be subdivided into three subgroups (I had not started yet: $N=20$; I have done my first tries: $N=28$; I reversed to my prior diet: $N=16$ ), ANOVAs were conducted. No significant between-subgroups differences were found for vulnerability $(p=.56)$, pros $(p=.58)$, cons $(p=.11)$, procrastination $(p=.40)$, and self-efficacy $(p=.07)$.

All social-cognitive variables were correlated weakly or moderately (absolute values of $r$ ranged from .09 to .43). Positive and negative outcome expectancies were unrelated. The strongest associations were found between self-efficacy and cons $(r=-.37, p<.001)$ and between vulnerability and pros $(r=-.43, p<.001)$. Descriptive statistics for all variables are displayed in Table 1.

To test discontinuity patterns, polynomial trend analyses were performed regarding Stages 2, 3, 5 of the PAPM and Stages $6 \mathrm{a}$ and $6 \mathrm{~b}$. Stage 4 of the PAPM was not included in the trend analyses because the sequence to action excludes the decision not to act. Linear trends were significant for all variables except for procrastination (see Table 2). Quadratic trends are significant for all variables. A significant cubic trend was found for pros. For pros and self-efficacy, linear trend effects were stronger in size than effects of quadratic and cubic trends. For vulnerability, cons, and procrastination, the quadratic 
Table I. Descriptive statistics for social-cognitive variables

\begin{tabular}{lccccc}
\hline & Vulnerability & Pros & Cons & Self-efficacy & Procrastination \\
\hline$M$ & 2.91 & 2.41 & 1.78 & 3.03 & 2.70 \\
SD & 1.18 & 0.65 & 0.60 & 0.72 & 0.52 \\
Scale range (Mean scores) & $1.00-6.00$ & $1.00-4.00$ & $1.00-4.00$ & $1.00-4.00$ & $1.00-4.00$ \\
\hline
\end{tabular}

Table 2. Results of polynomial trends analyses (weighted) for Stages 2, 3, 5, 6a, 6b

\begin{tabular}{|c|c|c|c|c|c|c|}
\hline \multirow[b]{3}{*}{ Social-cognitive variables } & \multicolumn{6}{|c|}{ Trends } \\
\hline & \multicolumn{2}{|c|}{ Linear } & \multicolumn{2}{|c|}{ Quadratic } & \multicolumn{2}{|c|}{ Cubic } \\
\hline & $F$ & $p$ & $F$ & $p$ & $F$ & $p$ \\
\hline Vulnerability & 57.97 & $<.01$ & 78.22 & $<.01$ & 0.08 & .78 \\
\hline \multicolumn{7}{|l|}{ Outcome expectancies } \\
\hline Pros & 67.02 & $<.01$ & 9.84 & $<.01$ & 4.84 & .03 \\
\hline Cons & 15.57 & $<.01$ & 30.06 & $<.01$ & 0.52 & .47 \\
\hline Self-efficacy & $|27.5|$ & $<.01$ & 55.56 & $<.01$ & 0.01 & .96 \\
\hline Procrastination & 1.97 & .16 & 25.82 & $<.01$ & 1.19 & .28 \\
\hline
\end{tabular}

Note. Highest $F$ statistics in bold.

trends were the strongest in size. Thus, the results of the trend analyses indicated discontinuity patterns for vulnerability, negative outcome expectancies, and procrastination.

To examine change patterns of social-cognitive measures across the stages, an ANOVA was performed for all stages, including Stage 4 (decided not to act). Stage 4 was included to examine the differences between the previous stage, deciding about action, and an alternative stage, decided to act. Simple contrasts and global effects were used to test the effects of social-cognitive variables across the stages. Table 3 displays significant differences across the stages for the social-cognitive variables.

\section{Vulnerability}

Significant differences between the stages were found for vulnerability, $F(5,802)=38.74, p<.01$ (see Fig. 1). The general patterns for vulnerability represent quadratic trends (see Table 2). Participants classified as unengaged by the issue perceived lower vulnerability than those deciding about action and those who had decided to act (see Table 3). Respondents who decided not to act did not differ significantly from those who were making their decisions and those who had already decided to act. After an individual had decided to act, the potential risk perception was reduced across subsequent stages (see Fig. 1). Participants who are acting with intention of maintenance feel less exposed to potential risk and perceive the lowest vulnerability. The results of the analyses for perceived vulnerability are consistent with stage model assumptions. As hypothesized, participants who were assigned to decision stages 
Table 3. Variables that discriminate between pairs of PAPM stages (Contrasts by means of $t$ tests)

\begin{tabular}{|c|c|c|c|c|c|}
\hline PA.PM stages & Deciding about action & Decided not to act & Decided to act & $\begin{array}{c}\text { Acting with no intention } \\
\text { of maintenance }\end{array}$ & $\begin{array}{c}\text { Acting with intention about } \\
\text { maintenance }\end{array}$ \\
\hline \multirow[t]{4}{*}{ Unengaged by issue } & Vulnerability ${ }^{* * *}$ & Vulnerability** & Vulnerability ${ }^{* * *}$ & Vulnerability ${ }^{\dagger}$ & Vulnerability*k \\
\hline & Cons* & Pros* & Pros*; Cons** & Pros** & Pros \\
\hline & Self-efficacy ${ }^{\dagger}$ & Self-efficacy* & Procrastination** & Self-efficacy ${ }^{\dagger}$ & Self-efficacy*k \\
\hline & Procrastination** & Procrastination $^{\dagger}$ & & Procrastination** & \\
\hline \multirow[t]{4}{*}{ Deciding about action } & & Pros*** & & Vulnerability*** & Vulnerability** \\
\hline & & Procrastination $^{\dagger}$ & & $\operatorname{Pros}^{\dagger}$ & Pros**; Cons** \\
\hline & & & & Self-efficacy** & Self-efficacy** \\
\hline & & & & & Procrastination** \\
\hline \multirow[t]{3}{*}{ Decided not to act } & & & Pros** & Vulnerability** & Vulnerability** \\
\hline & & & Cons $^{\dagger}$ & Pros** & Pros**; Cons** \\
\hline & & & Procrastination* & Self-efficacy ${ }^{* *}$ & Self-efficacy** \\
\hline \multirow[t]{4}{*}{ Decided to act } & & & & Vulnerability* & Vulnerability ${ }^{* *}$ \\
\hline & & & & Cons*** & Pros**; Cons** \\
\hline & & & & Self-efficacy ${ }^{* * *}$ & Self-efficacy ${ }^{* * *}$ \\
\hline & & & & & Procrastination $* *$ \\
\hline \multirow{4}{*}{$\begin{array}{l}\text { Acting with no intention } \\
\text { of maintenance }\end{array}$} & & & & & Vulnerability** \\
\hline & & & & & Procrastination $* *$ \\
\hline & & & & & Self-efficacy** \\
\hline & & & & & Pros**; Cons** \\
\hline
\end{tabular}

Note. ${ }^{\dagger} p<.10 ; * p<.05 ; * * p<.01$ 


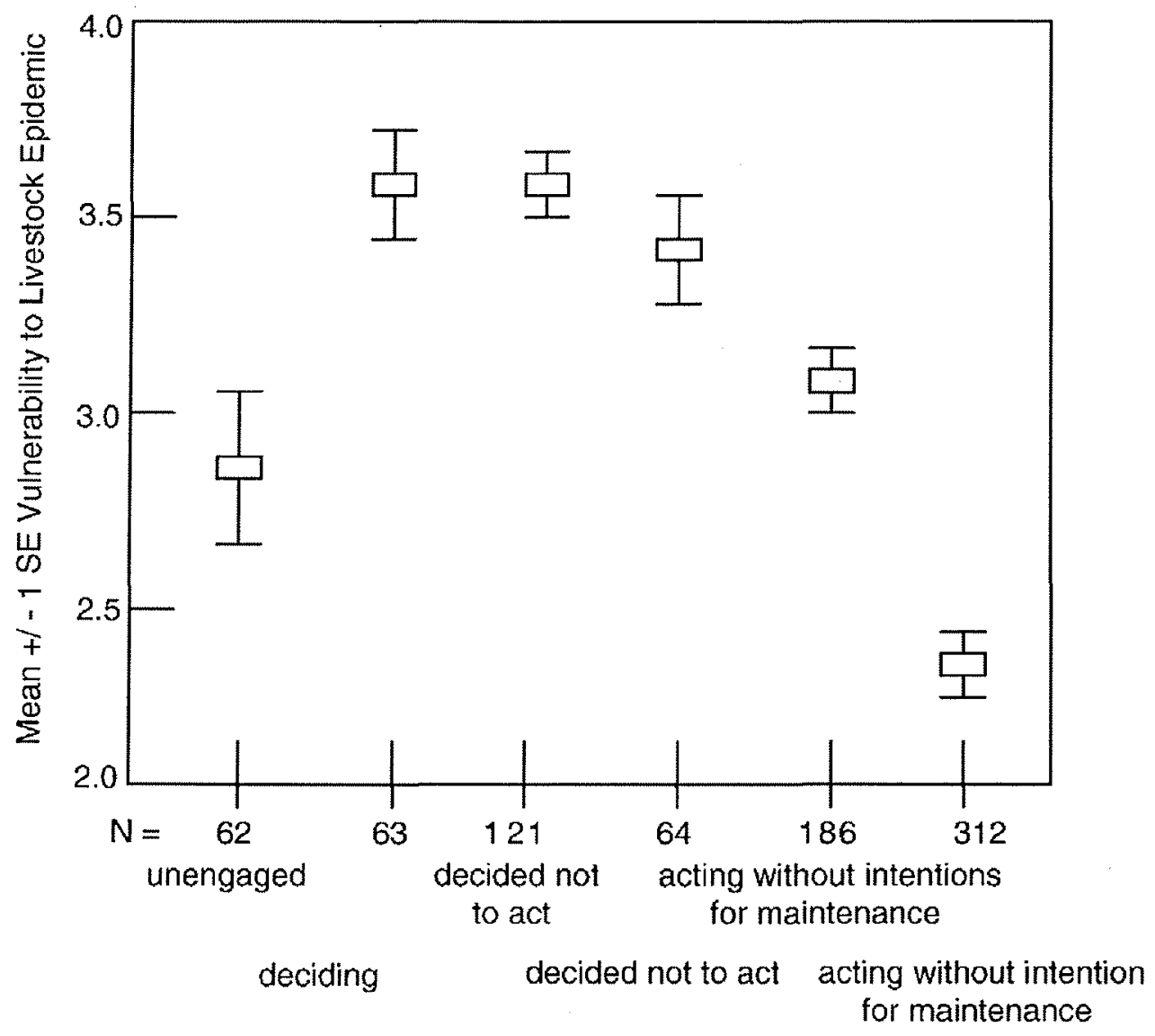

Figure I. Mean values and standard errors of vulnerability.

perceived higher vulnerability than those who were unengaged and those in action stages (see Table 3).

\section{Outcome expectancies}

The positive outcome expectancies differed significantly between stages, $F(5,802)=34.81, p<.01$ (see Fig. 2). As hypothesized, participants from earlier stages of behaviour change had lower positive outcome expectancies than those assigned to action stages (see Table 3). Participants from early stages expected fewer pros than those from later stages, with the exception of Stage 4. Participants who decided not to act expected significantly less pros than participants assigned to all other stages. The overall continuity pattern for pros was in line with a pseudo-stage model. Expectations regarding positive outcomes did not differ in participants assigned to the stages decided to act and acting with no intention of maintenance. Participants in Stage $6 a$ perceived fewer pros than those in Stage 6b (see Table 3). Those who did not explicitly intend to act in the long term might expect fewer pros than those who intend to maintain the actions.

Negative outcome expectancies differed significantly across the stages, $F(5,802)=9.78, p<.01$. These changes formed discontinuity patterns (see Fig. 2). As hypothesized, cons were higher in participants deciding about action who decided to act than in respondents unengaged by the issue. Persons deciding about action and who decided not to act did not differ significantly from those who decided to act (see Table 3). Participants acting with no intention to maintain the actions expected significantly fewer negative consequences than participant who decided to act (Stage 5). The lowest 

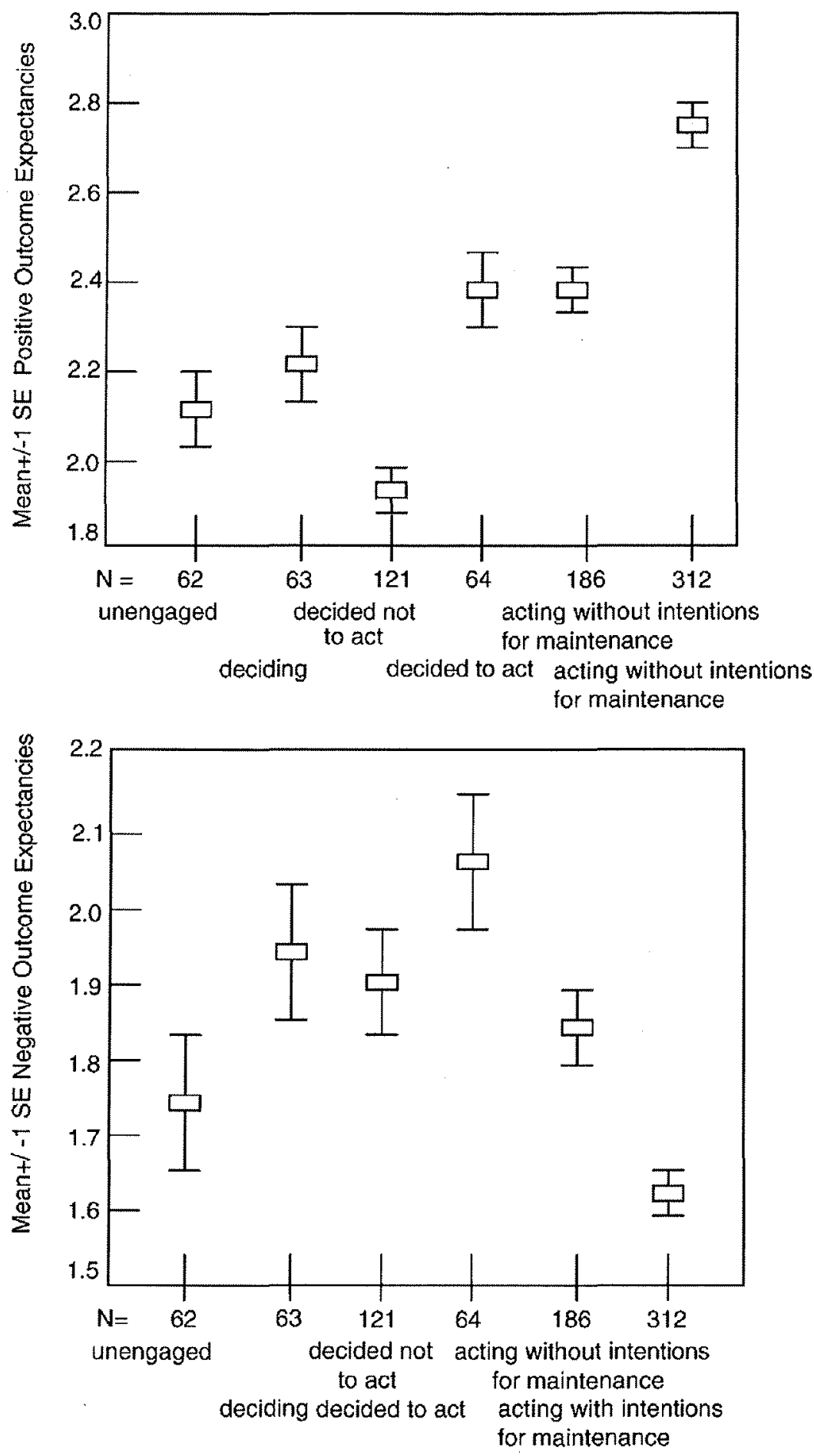

Figure 2. Mean values and standard errors of pros and cons.

negative outcome expectancies have been found in participants who were acting and intended to continue reduced meat consumption.

\section{Self-efficacy}

Optimistic self-beliefs about the personal ability to maintain the actions differed significantly across stages, $F(5,802)=50.29, p<.01$. Self-efficacy increased in a linear manner, beginning from Stage 3 to Stage $6 \mathrm{~b}$ (see Fig. 3). The individuals in actional stages differed significantly from those who were assigned to decision stages (see Table 3). Participants with an intention to maintain the action in the future reported stronger selfefficacy beliefs than persons in all other stages. 

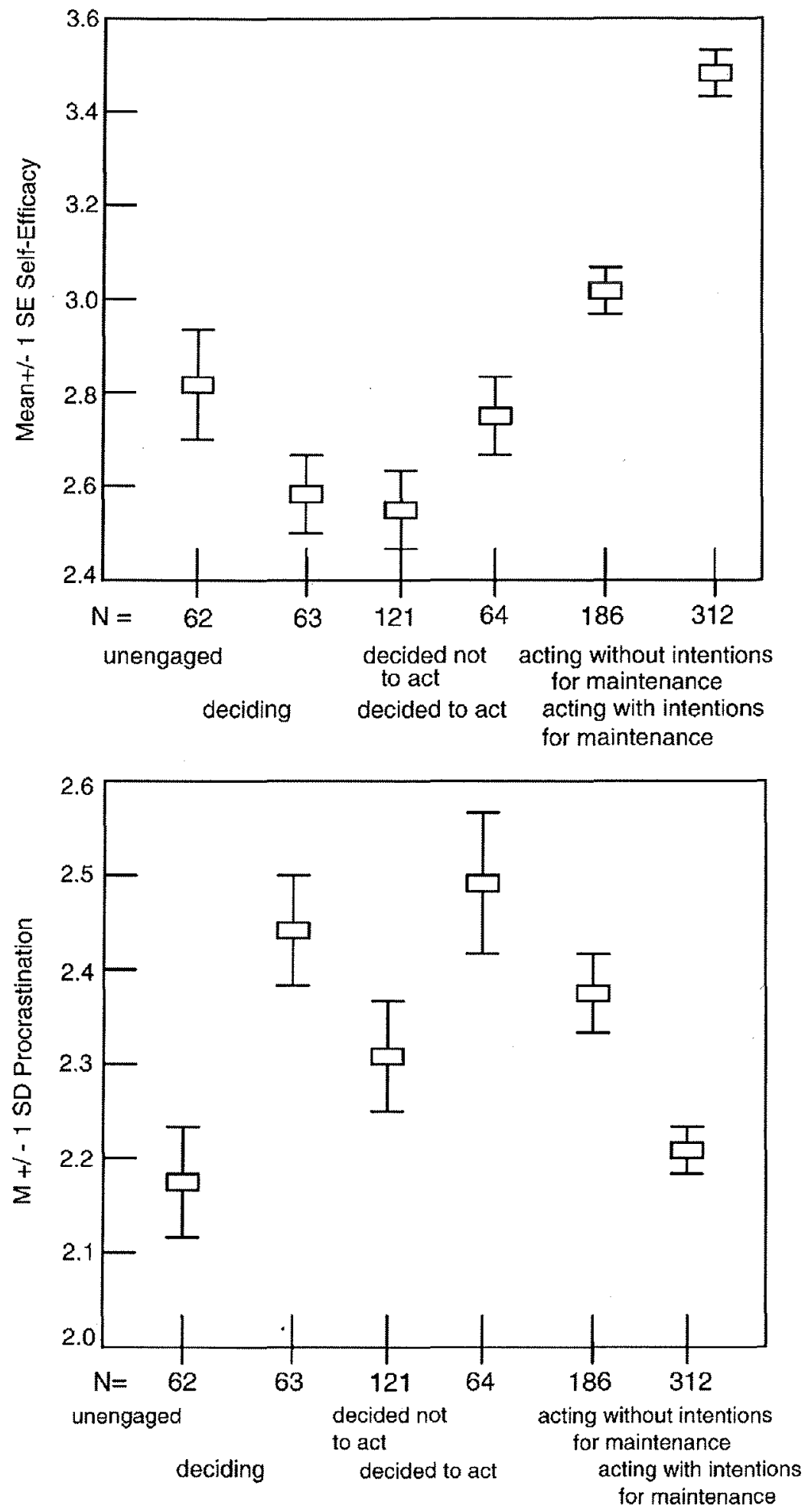

Figure 3. Mean values and standard errors of self-efficacy and procrastination.

Results of the trend analysis suggested that changes in self-efficacy formed rather linear trends than quadratic or cubic ones (see Table 2). However, a closer look at the results of the ANOVA indicates some possible discontinuity patterns, if only pre-actional stages are taken into account. Unengaged participants reported stronger self-efficacy beliefs than persons decided not to act (see Table 3). Excluding the participants from actional stages ( $6 a$ and $6 b$ ) resulted in a non-significant linear trend, $F(1,306)=0.70$, $p=.40$, and a significant quadratic trend, $F(1,306)=4.93, p=.03$. Therefore, in preactional stages, discontinuity patterns were observed for self-efficacy. 


\section{Procrastination}

For procrastination, significant differences between the stages were found, $F(5,802)=5.76, p<.01$ (see Fig. 3). Procrastination was high amongst those who were currently making up their mind and among those who decided to act but had not started yet to take action (see Table 3). Respondents who behaved in accordance to their intentions (Stages 2, 4, and 6) scored lower on the procrastination scale. Participants who were acting with no intention of maintenance might have a stronger procrastination tendency than persons who were acting with an intention about maintenance (see Table 3).

\section{Discussion}

The present study provides partial evidence for distinct stages of health behaviour change indicated by discontinuity patterns of social-cognitive variables over the stages. Regarding the stages of PAPM that were investigated, it was found that respondents who were assigned to Stage 2 (unengaged) perceived lower vulnerability and had lower procrastination as well as stronger confidence in their ability to reduce meat consumption than those in Stage 3 (deciding) or in Stage 4 (decided not to act). Unengaged participants expected less cons than those who were deciding. Those who decided not to act perceived fewer advantages of health behaviour change than persons in all other stages. Participants who decided to act anticipated more pros than persons who were unengaged and who had decided not to act. Regarding the two additional stages included in the study, some considerable differences were found. Participants acting with future intentions perceived the lowest vulnerability and the highest positive outcome expectancies. Persons acting without future intentions perceived lower vulnerability and less cons but higher self-efficacy compared with participants who decided to act.

The discontinuity patterns, obtained in the present study support the hypothesis that health behaviour change may be subdivided into stages. The patterns found for vulnerability confirm the role of risk perception in developing an intention to initiate action. Individuals who were unengaged perceived low vulnerability. Maintenance of initiated behaviour is also related to low perceived vulnerability, because those who maintain their diet expect risk-reducing effects of their actions. Procrastination was high in persons deciding about action, and also in those who were decided to act but had not yet initiated the action. Therefore, the decision process forms a separate stage that might be distinguished from being unengaged by the issue and stages of acting. Respondents deciding about action and those who decided to act, but not yet acting might be characterized as individuals who have difficulties with initiating behaviour change. These are individuals who are stuck in the process of decision-making or individuals who have already made up their mind, but do not have enough self-regulatory skills to initiate the action.

Self-efficacy and positive outcome expectancies were associated with the stages in a linear way providing support for a pseudo-stage model. A linear increase across the stages for positive outcome expectancies was also found in studies supporting the TTM (Rosen, 2000). Positive outcome expectancies might increase across stages. The positive effects of changes in nutrition might be observed a long time after the initiation. Those individuals who acted without an intention to maintain expected fewer positive outcomes than persons who acted with an intention to maintain their diet. The results 
for self-efficacy are ambiguous. Trend analyses for all stages excluding the Stage 4 (decided not to act) revealed continuity patterns, but an additional trend analysis for the pre-actional stages indicated some discontinuity patterns. As long as individuals are unengaged, self-efficacy may be less important than in later stages when individuals initiate their actions. Similar findings for perceived control over behaviour were obtained in a study testing the TTM (Armitage \& Arden, 2002). Change patterns for selfefficacy in both studies support the assumption of a sequence of motivational processes in which risk perception and outcome expectancies develop prior to self-efficacy beliefs (Schwarzer, 1999; Weinstein, 1988).

The findings of this study indicate continuity patterns in some social-cognitive variables (e.g. pros), that are congruent with theories. This raises questions regarding the selection of variables in the testing of stage models. Some variables might not allow empirical support for differentiating between stages, because their importance increases continuously as individuals move from pre-actional to actional stages. To provide further support for stage models it is desirable to select social-cognitive variables that do not show a linear increase or decrease across stages, but allow differentiation between stages due to their specific importance in selected stages only. Future studies that aim at testing stage models should incorporate variables such as phase-specific self-efficacy (Luszczynska \& Schwarzer, 2003) and planning (Schwarzer, 2001).

Some limitations of stage assignment might be addressed. The PAPM does not provide a rule regarding the stage assignment of individuals who have tried to act but failed. In the present study, the three subgroups assigned to Stage 5 did not differ in terms of social-cognitive variables. This may result from small size of subsamples. Other models suggest that individuals who have tried and failed are in a distinct stage (cf. Lippke, Sniehotta, \& Luszczynska, in press).

Further problems with the stage assignment arise if continuous behaviours are under investigation. For example, individuals who reduced their meat consumption, but not as much as they intended, might be assigned to either decisional stage or action/maintenance stage. The assignment depends on a researcher's decision: an arbitrarily set cut-off score on a target behaviour continuum. Future research should test the consequences of the employment of different cut-off scores on the stage assignment.

The present study has several limitations. Reliability of data collection from the Internet might be addressed. Studies that address effects of data collection showed that employment of an on-line-questionnaire and an interview resulted in the same frequencies of health behaviours (cf. Rhodes, DiClemente, Cecil, Hergenrather, \& Yee, 2002). A cross-sectional design allows the assignment of participants to stages, but the process of health behaviour change across stages cannot be tested. Further limitations are due to the unequal distribution of participants across stages, and the relatively low number of participants classified as unengaged or decided not to act. Exclusion of two stages of the PAPM (unaware of issue and maintenance) do not allow for an acid test for the entire model. The present study relies on self-report measures that may be of limited validity (Stone \& Shiffman, 2002). A cross-sectional design is usually accepted as sufficient to test stage models (Sutton, 2000; Weinstein, Lyon, et al., 1998, Weinstein, Rothman, et al., 1998). Further studies employing an experimental design might investigate whether differences in social-cognitive variables are causes or consequences of stage transitions. Longitudinal and experimental studies are required to test discontinuity patterns in groups of persons who transit across stages during the time intervals between the measurements. In order to provide more evidence for the 
usefulness of stage models, further studies should examine discontinuity patterns in other stage models. The results of the present study suggest that two groups of acting persons might be distinguished, that is, individuals acting with no intention of maintenance and individuals acting with the intention of maintenance. The persons who intended to maintain their actions in the future differed in social-cognitive variables from those who were acting but were undecided about future maintenance. The lack of intention to maintain low meat consumption might refer to the reaction of European meat consumers who were exposed to the information on BSE during the year 2001. Adopting temporary precautionary actions before a final intention is formed can be an adaptive response regarding a new perceived risk. Further studies are needed to validate the suggested distinction in the context of other precautionary actions.

\section{Acknowledgements}

The authors would like to thank James E. Maddux and Ralf Schwarzer for their helpful comments.

\section{References}

Ajzen, I. (1991). The theory of planned behavior. Organizational Bebavior and Human Decision Processes, 50, 179-211.

American Association for the Advancement of Science (1999). Etbical and legal aspects of buman subjects research on the Internet. Retrieved March 12, 2000, from http://www.aaas.org/spp/ sfrl/projects/intres/report.pdf.

Armitage, C. J., \& Arden, M. A. (2002). Exploring discontinuity pattern in the transtheoretical model: An application of the theory of planned behaviour. British Journal of Healtb Psychology, 7, 89-103.

Blalock, S. J., DeVellis, R. F., Giorgino, K. B., DeVellis, B. M., Gold, D., Dooley, M. A., Anderson, J. B., \& Smith, S. L. (1996). Osteoporosis prevention in premenopausal women: Using a stage model approach to examine the predictors of behaviour. Health Psychology, 15, 84-93.

Bruce, M. E., Will, R. G., Ironside, J. W., McConnell, I., Drummond, D., Suttie, A., McCardle, L., Chree, A., Hope, J., Birkett, C., Cousens, S., Fraser, H., \& Bostock, C. J. (1997). Transmissions to mice indicate that new variant CJD is caused by BSE agent. Nature, 389, 498-501.

Clemow, L., Stoddard, A. M., Costanza, M. E., Haddad, W. P., Luckmann, R., White, M. J., \& Klaus, D. (2000). Underutilizers of mammography screening today: Characteristics of women planning, undecided about and not planning a mammogram. Annals of Behavioural Medicine, 22, 80-88.

Ferguson, N. M., Ghani, A. C., Donnelly, C. A., Hahenaars, T. J., \& Anderson, R. M. (2002). Estimating the human health risk from possible BSE infection of the British sheep flock. Nature, 415, 420-424.

German Central Agency for Market and Price Reports (2001). Special report on BSE based on GfK domestic panel. Bonn, Germany: Zentrale Markt- und Preisberichtstelle GmbH.

Lee, C. (1993). Attitudes, knowledge, and stages of change: A survey of exercise patterns in older Australian women. Health Psychology, 12, 476-480.

Lippke, S., Sniehotta, F. F, \& Luszczynska, A. (in press). Social cognitions across the stages of behavior change. A comparison of two stage models. Polish Psychology Bulletin.

Luszczynska, A., \& Schwarzer, R. (2003). Planning and self-efficacy in the adoption and maintenance of breast self-examination: A longitudinal study on self-regulatory cognitions. Psychology and Health, 18, 93-108.

Prochaska, J. O., \& DiClemente, C. C. (1982). Transtheoretical therapy: Toward a more integrative model of change. Psychotherapy: Theory, Research, and Practice, 19, 275-288. 
Prusiner, S. B. (1997). Prions. Les prix nobel 1997 (pp. 262-323). Stockholm, Sweden: Nobel Foundation.

Rhodes, S. D., DiClemente, R. J., Cecil, H., Hergenrather, K. C., \& Yee, L. J. (2002). Risk among men who have sex with men in the United States: A comparison of an internet sample and a conventional outcome sample. AIDS Education and Prevention, 14, 41-50.

Rosen, C. S. (2000). Integrating stage continuum models to explain processing of exercise messages and exercise initiation among sedentary college students. Health Psychology, 19, $172-180$.

Schwarzer, R. (1999). Self-regulatory processes in the adoption and maintenance of health behaviours. The role of optimism, goals, and threats. Journal of Health Psychology, 4 , 115-127.

Schwarzer, R. (2001). Social-cognitive factors in changing health-related behavior. Current Directions in Psychological Science, 10, 47-51.

Schwarzer R., Schmitz, G., \& Diehl, M (2000). Procrastination Scale. Retrieved January 20, 2001, from www.coping.de.

Stone, A. A., \& Shiffman, S. (2002). Capturing momentary, self-report data: A proposal for reporting guidelines. Annals of Bebavioral Medicine, 24, 236-243.

Sutton, S. (1998). Predicting and explaining intentions and behavior: How well are we doing? Journal of Applied Social Psychology, 28, 1317-1338.

Sutton, S. (2000). Interpreting cross-sectional data on stages of change. Psychology and Healtb, 15, 163-171.

Tice, D. M., \& Baumeister, R. F. (1997). Longitudinal study of procrastination, performance, stress, and health: The costs and benefits of dawdling. Psycbological Science, 8, 454-458.

Weinstein, N. D. (1988). The precaution adoption process. Health Psychology, 7, 355-386.

Weinstein, N. D., Lyon, J. E., Sandman, P. M., \& Cuite, C. L. (1998). Experimental evidence for stages of precaution adoption. Health Psychology, 17, 445-453.

Weinstein, N. D., Rothman, A., \& Sutton, S. R. (1998). Stage theories of health behaviour. Health Psycbology, 17, 1-10.

Weinstein, N. D., \& Sandman, P. M. (1992). A model of the precaution adoption process: Evidence from home radon testing. Health Psychology, 11, 170-180.

Weitkunat, R., Pottgiesser, C., Meyer, N., Crispin, A., Fisher, R., Schotten, K., Kerr, J., \& Ueberla, K. (2003). Perceived risk of bovine spongiform encephalopathy and dietary behavior. Journal of Health Psychology, 8, 373-381. 